\title{
Investigations on printed elastic resistors containing carbon nanotubes
}

\author{
Marcin Słoma • Małgorzata Jakubowska $\cdot$ Andrzej Kolek • \\ Krzysztof Mleczko $\cdot$ Piotr Ptak • Adam Witold Stadler • \\ Zbigniew Zawiślak $\cdot$ Anna Młożniak
}

Received: 19 August 2010/Accepted: 18 January 2011/Published online: 9 February 2011

(C) The Author(s) 2011. This article is published with open access at Springerlink.com

\begin{abstract}
This paper presents the results of authors investigations on elaboration of a new thick film composition filled with carbon nanotubes (CNTs). The polymer composition consists of polymer vehicle, which is the solution of organic resin in certain combination of solvents, and functional phase-carbon nanotubes well dispersed in the vehicle. The pastes were applied with screen-printing on several substrates and temperature cured. The properties of obtained layers were characterized. Series of samples were prepared with different amount of CNTs to evaluate electrical properties. Changes in resistance were investigated during periodic mechanical and temperature stresses, realized through cyclical bending and rapid temperature change. Tensometric effect was also investigated. Investigations have proved that polymer composites based on carbon nanotubes exhibit high resilience to stress factors. Resistance change in function of temperature was also investigated to evaluate temperature coefficient of resistance (TCR). All this aspects are important for elastic resistors fabrication in printed electronics microcircuits. Resistance and noise measurements in cryostats have also been involved. 1/f type noise has been observed. Noise
\end{abstract}

M. Słoma $(\bowtie) \cdot$ M. Jakubowska

Institute of Metrology and Biomedical Engineering, Faculty of Mechatronics, Warsaw University of Technology,

Św. A. Boboli 8, 02-525 Warsaw, Poland

e-mail: marcin.sloma@mchtr.pw.edu.pl

M. Jakubowska · A. Młożniak

Institute of Electronic Materials Technology, Wólczyńska 133, 01-919 Warsaw, Poland

A. Kolek · K. Mleczko · P. Ptak - A. W. Stadler · Z. Zawiślak Department of Electronics Fundamentals, Faculty of Electrical and Computer Engineering, Rzeszów University of Technology, W. Pola 2, 35-959 Rzeszów, Poland intensity, calculated in decade frequency bands, rises significantly with increasing temperature. Activation energies of thermally activated noise sources (TANS) have been revealed using low-frequency noise spectroscopy. Relatively large value of negative TCR has been obtained from resistance versus temperature curve. Calculated dimensionless sensitivity is similar to that observed in cryogenic temperature sensors. However, bulk noise intensity of resistive layer is larger than obtained for lead containing $\mathrm{RuO}_{2}$ based resistive layers.

\section{Introduction}

Carbon nanotubes (CNTs) and fullerenes have attracted extensive attention in many branches of industry, since their discovery at the end of last century. Carbon nanotubes exhibit outstanding electrical and mechanical properties, that may revolutionize field of electronic elements technology. Addition of carbon nanotubes caused new properties of many materials, such as polymers, metals or carbon matrices by enhancing their strength and their thermal and electrical conductivity [1-4]. Nanotube-based composites improved structural materials for construction elements [5, 6], electrical conductors for special electronic applications [7, 8], probes and sensors for biotechnology $[9,10]$, and many other disciplines.

On the other hand, development of printed and flexible electronics technologies has been growing fast in the last few years. There has been growing interest in the development of various printing techniques for patterning electronics circuits. They could be used as a low-cost alternatives to current technologies in production of flexible electronics, where mechanical flexibility is required. They could also be applied for disposable electronics, 
where silicon chip packaging costs are too high. Potential applications for these circuits are flexible electronics [11], biochemical sensors [12], functional elements (i.e. printed transistors) [13, 14] or smart clothing [15]. The electronic circuits applied on flexible substrates such as polymer foil, paper, textiles are exposed to series bends. Therefore, there is a strong necessity of elaboration of new materials which will exhibit better mechanical properties.

Having in mind outstanding mechanical properties of CNT, the authors expected that application of carbon nanotubes to polymer thick film paste can provide thick film resistive and conductive layers, with better properties than these of standard graphite or carbon black compositions.

\section{Materials and preparation}

Nanostructured materials investigated by the authors in this research were commercially available CVD grown carbon nanotubes. Raman spectra from $2.5 \mathrm{~mW}$ laser beam (Fig. 1a) indicated that material is mostly of graphite structure ( $\mathrm{D}$ and $\mathrm{G}$ bands) what is common for multiwalled carbon nanotubes (MWCNT). Transmission electron microscopy (TEM) observations confirms this assumptions (Fig. 1b), and also allows to estimate mean length and mean diameter of nanotubes to be around $0.5-5 \mu \mathrm{m}$ and $20-40 \mathrm{~nm}$, respectively but longer nanotubes were also observed. While CNT material was used without additional purification process, catalyst and amorphous carbon are also observed.

Polymer vehicle consists of poly(methyl methacrylateco-butyl methacrylate) (PMMA-PBMA) copolymer as $34 \%$ butyl carbide acetate solution. With precisely controlled volume of nanotubes in polymer vehicle, we can obtain composite material that is electrically conductive with low sheet resistance, and suitable for screen printing process.

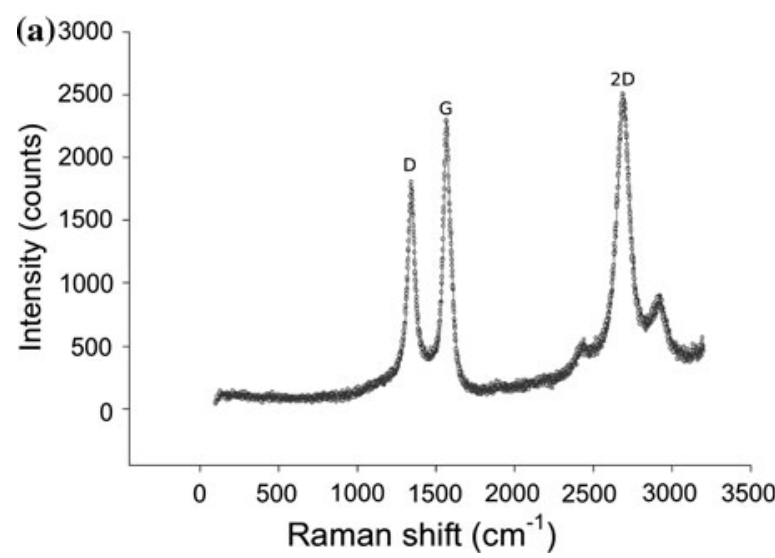

Fig. 1 a Raman spectra and b TEM micrograph of CNT material
While CNTs are very attractive for modern materials applications their nano-scale dimensions creates an obstacle for easy and effective addition to other materials, such as polymer resins. It is related to the poor solubility and processability $[16,17]$. Many methods were proposed to enhance their stirring properties. Some of them include surface activation and modification what may lead to undesirable changes in mechanic and electronic properties of CNTs [18]. On the other hand, obtaining homogeneous suspension is almost impossible through simple mixing of CNTs with liquids or solid particles. Creation of agglomerates occurs due to high bounding forces between separate CNTs [4]. Most common ways for mixing particles are ball grinding for solid state particles [5] and low energy ultrasonic mixing for liquid suspensions [17]. In both methods we can encounter problems with deagglomeration of nanoparticles, due to aforementioned high interparticle bounding forces. A novel method of mixing nanoparticles by rapid expansion of high-pressure suspensions [18] produces suspension mixtures with low amount of agglomerates, but it is time and quantity ineffective and needs high pressure apparatus.

Our goal was to adapt well known thick film composition preparation procedures, to provide simple, quick and low cost technology expected in fabrication of printed electronics devices. In our case simple mechanical mixing of corresponding materials with pestle and mortar resulted with unsatisfying mixtures, with high ratio of nanotube agglomerates of more than $250 \mu \mathrm{m}$ size, and low level of dispersion in polymer resin. Therefore solvent suspension of CNT was ultrasonically stirred in IS-1K ( $300 \mathrm{~W}, 30 \mathrm{kHz})$ ultrasonic bath for $1 \mathrm{~h}$, added to polymer base, and stirred for one more hour to obtain anticipated degree of dispersion. Last procedure was three roll milling with $100 \mu \mathrm{m}$ gap between the rollers for final homogenization. Two types of compositions were fabricated with 0.25 and $1 \mathrm{wt} \%$ of carbon nanotubes. From this compositions sample layers were fabricated,

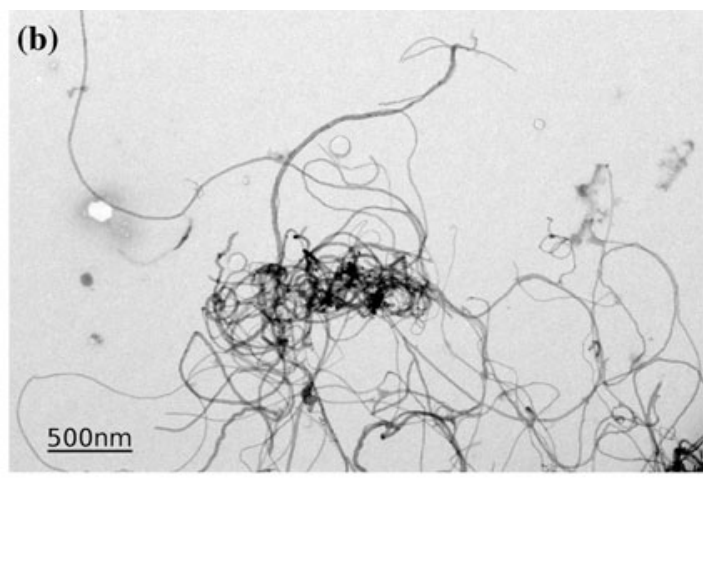



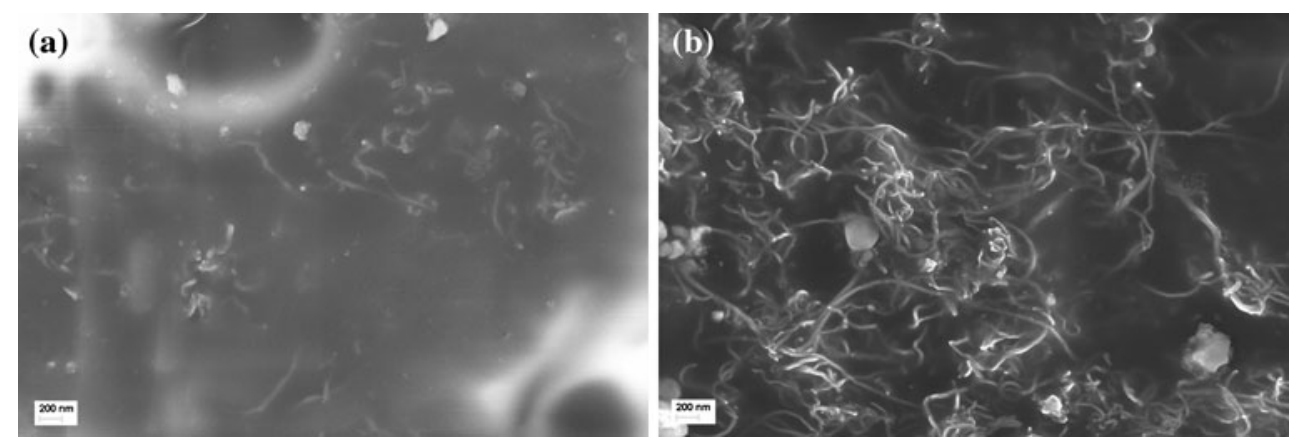

Fig. 2 Micrographs of obtained layers printed from compositions containing a 0.25 wt $\%$ and b 1 wt $\%$ CNTs

described later as $0.25 \mathrm{CNT}$ and $1 \mathrm{CNT}$ respectively. Micrographs from HRSEM observations of obtained composite layers are presented in Fig. 2.

For mechanical bending test, thermal shock test and high temperature thermal coefficient of resistance (TCR) measurements, nanotube-polymer compositions screen printed on polyester foil were used. Samples were cured at $130{ }^{\circ} \mathrm{C}$ for $1 \mathrm{~h}$ to evaporate solvent and harden polymer base. Specially designed pattern allows to obtain comparative sample paths with uniform length of $24 \mathrm{~mm}$ and different surface area. To unify electrical measurements, silver electrodes were screen printed to provide uniform current flow over whole area of sample. Test pattern (black) with silver electrodes (gray) and selected axis of bending is presented in Fig. 3a. For resistance measurements in relation to temperature change and noise properties, compositions were printed on alumina substrate, and cured the same way as samples printed on polyester. Samples were stripes of $L=15 \mathrm{~mm}$ length, $w=1 \mathrm{~mm}$ width, and $d=2.5 \mu \mathrm{m}$ thickness, with side terminals and silver electrodes. Pattern was designed specially for noise properties explorations in thick film resistors [19]. With regard to resistance values measured on cryogenic laboratory stand only $1 \mathrm{CNT}$ samples were used for further low temperature measurements. Printed $0.25 \mathrm{CNT}$ samples exhibit large resistance, hence only some selected

(a)
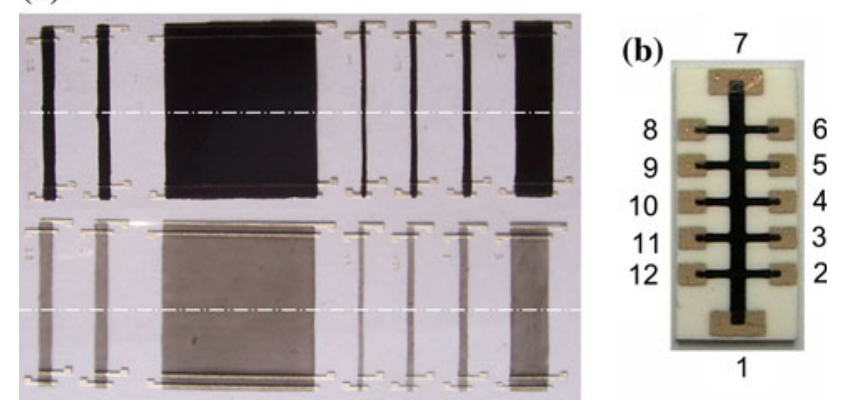

Fig. 3 Macroscopical pictures of obtained screen printed samples used for a mechanical resistance measurement and $\mathbf{b}$ noise measurements with terminals numeration measurement procedures could be applied, due to instruments limitations. Macroscopic picture with terminals numeration is presented in Fig. 3b.

Obtained printed layers containing carbon nanotubes were directly compared to traditionally used for such application polymer-graphite compositions. Commercially available Electrodag 421 SS (E421SS) composition from Acheson Industries Ltd. was used. Samples from graphite paste were fabricated with the same procedure as nanotube samples.

\section{Instruments and experimental}

Conducted experiments were focused on the investigations of mechanical fatigue and durability of the produced polymer composite, used afterwards as elastic circuits or elements. For that two types of mechanical tests were performed including cyclical bending test and singular bending test. Thermal shocks test and two types of electrical properties measurements in function of temperature change were also performed. No hardness test was performed such as indentation, because this parameter is not crucial for printed electronics.

\subsection{Noise measurements circuit}

Noise identification and noise level measurements were also conducted for resistive layers. For noise explorations, measurement setup previously used for thick-film resistors $[19,20]$, including dc bridge configuration, was adopted (see Fig. 4b). Selected pair of samples with matched resistance $R_{1-7} \equiv V_{1-7} / I$ in room temperature, where $V_{1-7}$ is the voltage between terminations 1 and 7 , and $I$ is biasing current (see Fig. 3b), inserted into bottom arms of the bridge, was biased from high voltage power supply unit through ballast resistors $R_{B}$ of large resistance $\left(R_{B} \gg R_{1-7}\right)$. Voltages from bridge diagonal and from selected subdiagonals were conditioned in differential low-noise preamplifiers, low-pass filtered and then were processed in Noise Signal Analyzer [21]. 
(a)

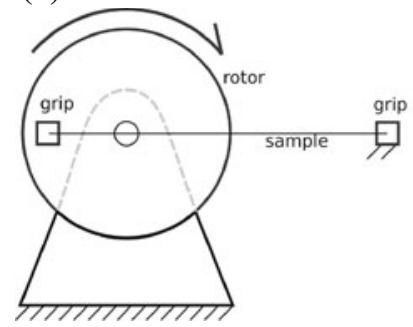

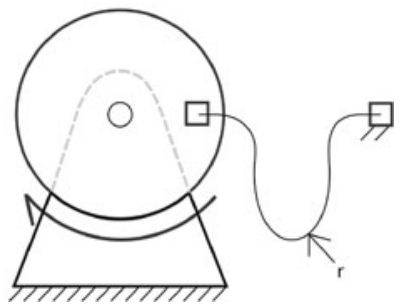

(b)

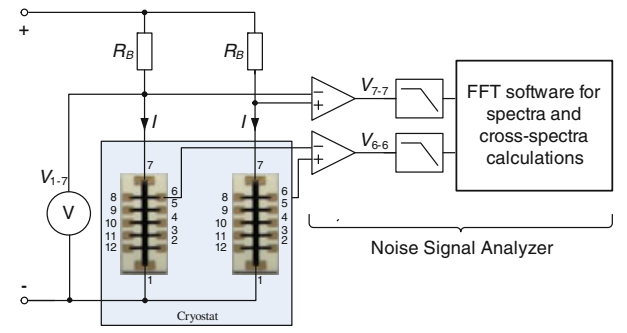

Fig. 4 a Schematic of the laboratory stand used for mechanical bending tests. b Measurement setup for low-frequency noise spectroscopy

FFT method was used to calculate in real-time spectra (for voltages from bridge diagonal) and cross-spectra for pairs of the voltages acquired from the bridge diagonal and one of the sub-diagonals. Time records of $2 \mathrm{~s}$ length with $524288=2^{19}$ samples, were used in continuous acquisition mode. Only low-frequency part of the averaged (over $10 \mathrm{~min}$ ) spectra, from $0.5 \mathrm{~Hz}$ up to $5 \mathrm{kHz}$, were recorded with the resolution of $0.5 \mathrm{~Hz}$. Actual samples temperature and resistance were also recorded.

\subsection{Mechanical properties elaboration stand}

Electric resistance measurements for mechanical, thermal shocks and high temperature TCR tests were conducted with Keithley 2001 multimeter. Changes in electrical properties were indicating resilience to stress factors. Thermal shock test were conducted in Heraeus HT 7012 S2 thermal shock chamber. Temperature and time cycles were corresponding to the thermal shock test of electronic circuits norm EN62137. The thermal cycle was set to $125{ }^{\circ} \mathrm{C}$ and $-40{ }^{\circ} \mathrm{C}$ for upper and lower temperature, respectively, cycled equally in $1 \mathrm{~h}$ period with $5 \mathrm{~s}$ temperature shift from upper to lower value and opposite. No additional humidification or addition of aggressive atmospheres was implemented. Both mechanical bending tests were performed on specially adapted laboratory stand, schematically presented in Fig. 4a. Bent sample was permanently fasten to fixed grip on one side and to movable grip on other side. Movable grip caused a wrap around symmetry axis of the bent sample. Maximal curvature obtained this way was around $0.14 \mathrm{~mm}^{-1}$. Cyclical bending tests were performed with frequency 350 cycles per minute. To determine correlation between number of bending cycles and electrical resistance changes, measurements of the resistance were taken successively during the test to observe tendency.

Thermal stability tests were performed in specially adapted heating chamber with measuring probes. Samples were placed on heating plate with temperature controlled via thermocouple element. Resistance was measured with two needle electrodes connected to Keithley 2001 digital multimeter. Temperature and resistance measurement were correlated in time and registered on PC computer, thus providing $R=f(T)$ function diagram.

\section{Results and discussion}

First test performed on selected polymer-nanotube and polymer-graphite samples was thermal shock test. Resistance measurements were taken before test and after 225 cycles. Mean values of sheet resistance and direct comparison of resistance values before and after thermal tests are presented in Table 1.

Maximum difference in electrical resistance of samples was around $1.5 \%$ for $1 \mathrm{CNT}$ and $4 \%$ for $0.25 \mathrm{CNT}$ samples. In relation polymer-graphite samples counted resistance growth of almost half of the value which is significant difference and can be unacceptable for the long term use in high reliability circuits.

Periodical mechanical stress test was also performed on 0.25CNT, 1CNT and E421SS samples, subjected to mechanical stress directly after printing and hardening. Changes of resistance versus number of cycles were investigated. Resistance values measured during bending cycles for $0.25 \mathrm{CNT}, 1 \mathrm{CNT}$ and E421SS samples are presented in Fig. 5.

Mechanical stresses negatively affected conductance in all tested samples. Electrical resistance increased around $4 \%$ for $0.25 \mathrm{CNT}$ sample and $2 \%$ for 1CNT sample after 300,000 cycles, and more than $26 \%$ for E421SS samples after 200,000 cycles. Resistance growth was linear for all samples. It seems that mechanical stress have relatively

Table 1 Mean values of resistance changes for thermal shock samples

\begin{tabular}{lccc}
\hline Sample & 0 cycles & 225 cycles & $\Delta R(\%)$ \\
\hline $1 \mathrm{CNT}$ & $3.82 \mathrm{k} \Omega$ & $3.87 \mathrm{k} \Omega$ & 1.46 \\
$0.25 \mathrm{CNT}$ & $23.94 \mathrm{k} \Omega$ & $24.91 \mathrm{k} \Omega$ & 4.04 \\
E421SS & $837.2 \Omega$ & $1.19 \mathrm{k} \Omega$ & 42.8 \\
\hline
\end{tabular}


Fig. 5 Measured values of sheet resistance during mechanical stress tests

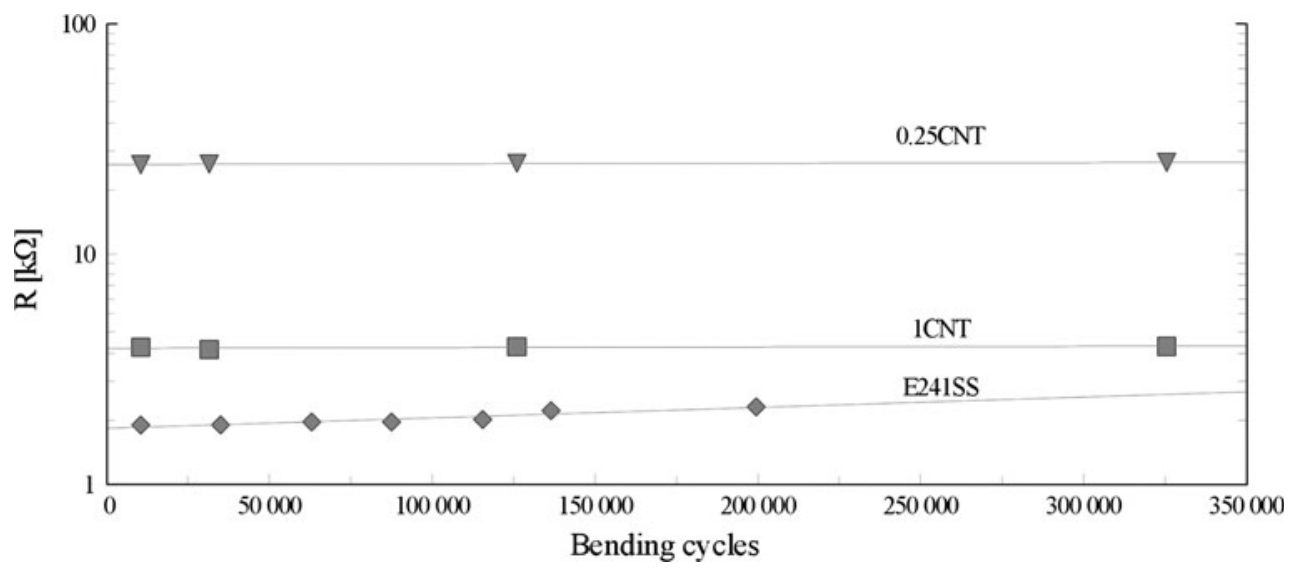

(a)

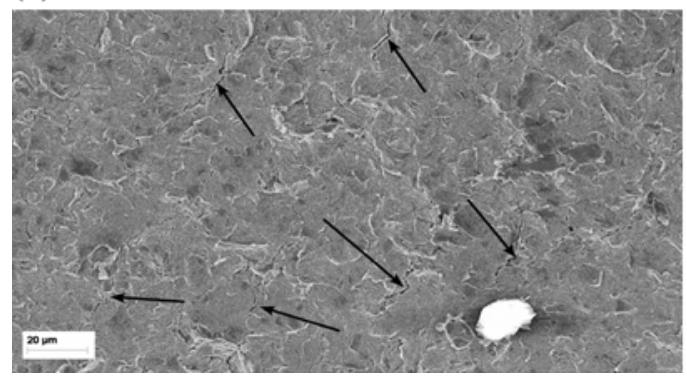

(b)

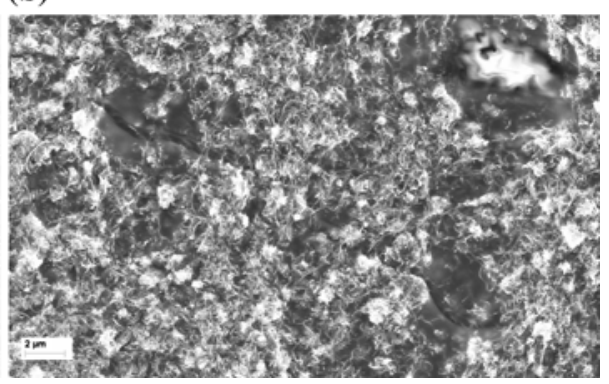

Fig. 6 Micrographs of a E421SS and b 1CNT samples after cyclical bending tests

low influence on polymer-nanotube layers. Nanotubes located in polymer base have very high length to diameter ratio which prevents contact loss between separate particles. Nanotubes have also high mechanical strength, and this kind of stress have no affect on their structure. SEM observations show microcracks in E421SS layers (see Fig. 6a), separating graphite grains, while there were no major cracks observed in 1CNT layer (see Fig. 6b).

Consecutive experiment was focused on resistance stability during curvature change of the samples. Unfortunately polymer paste application in elastic electronics is causing serious problem connected with tensometric effect. Mechanical stresses connected with stretching or bending of elastic polymer resistors can introduce dramatic resistance change, reaching few times of the initial value in case of rectangular shaped sensors. This phenomenon is caused by influence of conductive particles position change within elastic sensor structure, and depended directly on the elongation of the sensor body. Resistance changes in function of bending radius are presented in Fig. 7.

In this experiment the strong mechanical stress influence on resistance was detected for polymer-graphite layers. It is mostly effected by segmentation of active material area along the bending direction. While E421SS samples counted more than $60 \%$ change in resistance value, resistance of nanotube layers remained almost unchanged. This also indicates that CNT composites might be suitable for elastic electronics applications, while graphite composites properties instability caused by bending, may be far beyond acceptable level.

Measurements of thermal stability were conducted on two types of nanotube-polymer samples $(0.25 \mathrm{CNT}$ and 1CNT) and graphite-polymer sample E421SS. All samples were elaborated directly after printing and hardening. Resistance versus temperature measurements taken during experiment in $20 \mathrm{~s}$ period for $0.25 \mathrm{CNT}$ and $1 \mathrm{CNT}$ samples are presented in Fig. 8. After this test samples were left to cool down to room temperature and tested again with the same procedure. Samples after second temperature cycle are called 0.25CNT-T, 1CNT-T and E421SS-T, respectively.

Observed changes in resistance of polymer-nanotube samples were from 17 to $19 \%$ for 1CNT samples, and from 10 to $12 \%$ for $0.25 \mathrm{CNT}$ samples. Local fluctuation of resistance observed around $50{ }^{\circ} \mathrm{C}$, as well as hysteresis, are caused by local reorientation of polymer chains near $T_{\mathrm{g}}$ (glass transition) temperature of polymer base. This local effect in polymer is causing decrease of probability of nanotube-nanotube connection, lowering volume conductance in layer, and creating initial resistance growth. After second heat up this effects are much smaller, or even 
Fig. 7 Measured values of sheet resistance during bending tests

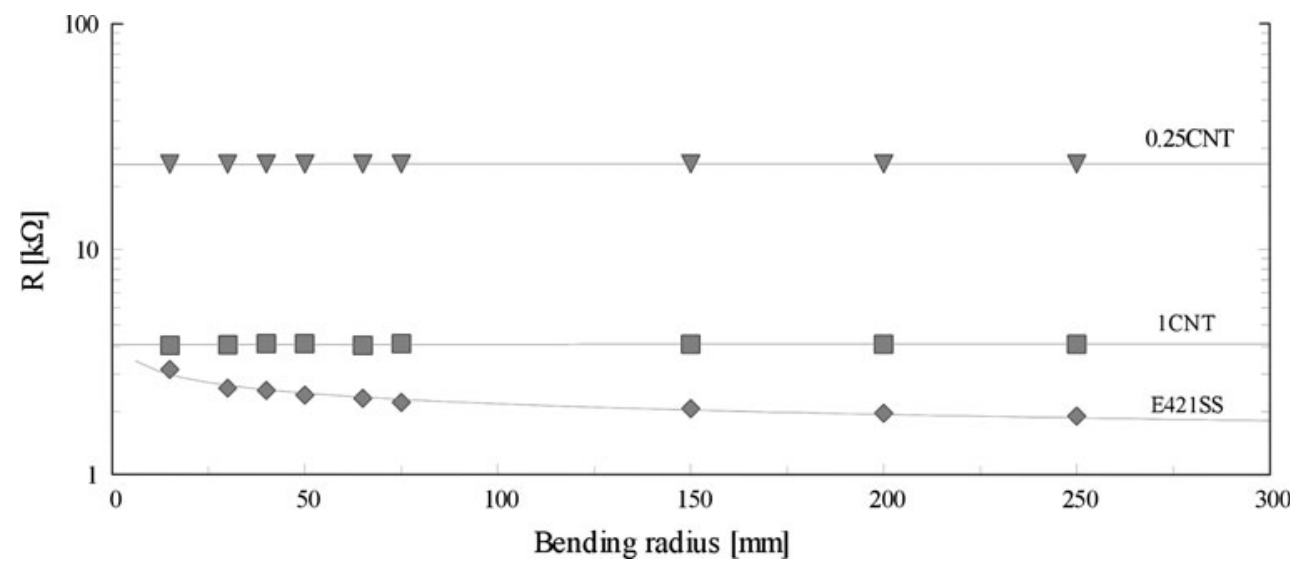

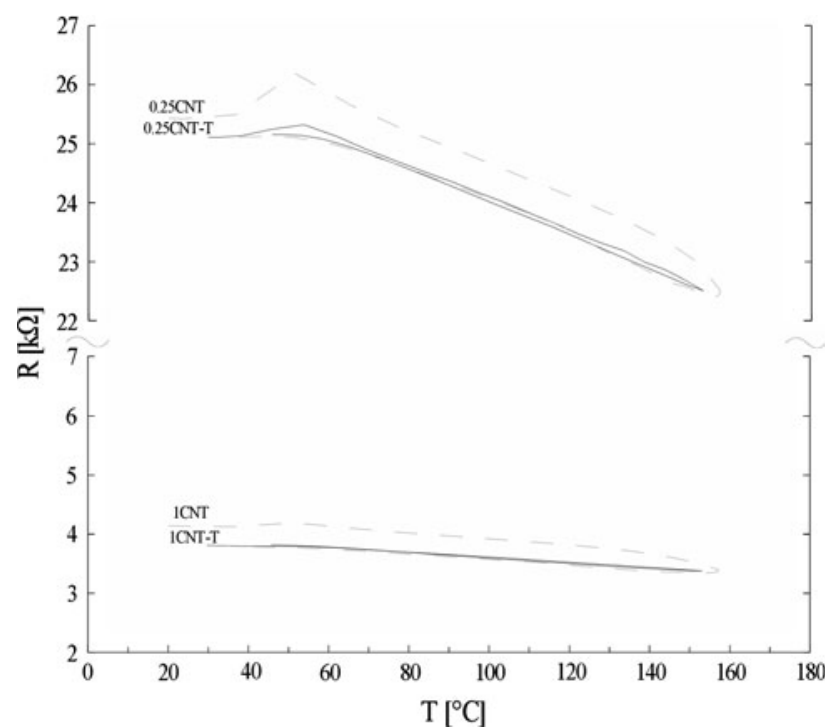

Fig. 8 Resistance values in function temperature change of polymernanotube printed resistors

unnoticeable, than for primary samples, what confirms assumptions about polymer chains reorientation.

Noise spectra shape and their dependence on excitation voltage were examined, to identify the noise. Power spectral densities $S_{V}$ of voltage fluctuations $\delta V$, measured on terminations 7 , in room temperature for several bias voltages $V_{1-7}$ are shown in Fig. 9a. Product of $S_{V}$ and frequency averaged in frequency bands versus sample's bias voltage is shown in Fig. 9b. Data collected in Fig. 9a and $\mathrm{b}$ indicate that the noise observed in studied samples in room temperature is $1 / \mathrm{f}$ noise induced by resistance fluctuations.

Temperature dependencies of resistance and noise were measured in liquid nitrogen cryostat in temperature range from $80 \mathrm{~K}$ up to $300 \mathrm{~K}$, and in liquid helium cryostat in temperature range from $5 \mathrm{~K}$ up to $80 \mathrm{~K}$. While temperature in cryostat was slowly rising, averaged spectra, sample's resistance and temperature were recorded continuously.
Temperature dependence of resistance $R_{1-7}$ is shown in Fig. 10a. In studied temperature range TCR changes its value from $-680 \mathrm{ppm} / \mathrm{K}$ at $T=300 \mathrm{~K}$ to $-0.162 \mathrm{~K}^{-1}$ at $T=5 \mathrm{~K}$ (see Fig. 10b). Dimensionless sensitivity, $A \equiv(T / R)(|\mathrm{d} R / \mathrm{d} T|)$, was also calculated in order to evaluate possibility of application of studied resistor as cryogenic temperature sensor. Calculated values of $A$ (see Fig. 10b), in the range from 0.18 to 0.88 , are typical for RTD cryogenic sensors [22].

Low-frequency noise spectroscopy (LFNS) relates to investigations of temperature dependence of low-frequency part of the spectra. Using data recorded during LFNS experiment, noise intensity, $S_{7-7}=\left\langle f_{S_{7-7}}\right\rangle_{\Delta f}$, was calculated, where $s_{7-7}=\left(S_{V}-S_{V=0}\right) / V_{1-7}^{2}$ is relative excess noise for terminations $1-7$, and averaging \langle\rangle was performed in frequency band $\Delta f$. Noise intensity significantly rises with temperature increase (see Fig. 11a). The observed behaviour is different from that in typical thickfilm resistors, where noise intensity is nearly constant for $T>10 \mathrm{~K}[19,20]$.

Noise map, i.e. the plot of the product $f_{s_{7-7}} \mathrm{~T}^{-1}$ versus frequency and temperature, prepared for the data from LFNS experiments, is shown in Fig. 11b. Applied method of noise scaling, was used in order to compensate temperature dependence of noise intensity and expose spectra components other than 1/f background noise. Reciprocal temperature scale helps in immediate identification of thermally activated noise sources (TANSs) visible as streaks on the map. Using Arrhenius plot, activation energies of TANSs from Fig. $11 \mathrm{~b}$ were calculated: $24.6 \mathrm{meV}$ for TANS observed in temperature range from 15 to $25 \mathrm{~K}$, and $0.67 \mathrm{eV}, 0.45 \mathrm{eV}, 1.1 \mathrm{eV}, 1.6 \mathrm{eV}$ for TANSs detected in the range $80-300 \mathrm{~K}$. However, in the latter temperature range number of TANSs is the largest, what is important with regard to applications in commercial temperature range. It is worth noting, TANS with similar values of activation energies were observed in thick film resistors [23]. 
Fig. 9 a Power spectral density $S_{V}$ of voltage fluctuations $\delta V$, measured on terminations 7 , in room temperature at different bias voltages $V_{1-7}$, listed in the legend. All data series but diamonds refer to $1 \mathrm{CNT}$. Diamonds are for $0.25 \mathrm{CNT}$. Solid line indicating pure $1 / \mathrm{f}$ noise has been added for reference. b Product of $f S_{V}$ versus excitation voltage $V_{1-7}$. Solid line shows linear dependence on $V_{1-7}$ square

Fig. 10 a Resistance versus temperature curve for $1 \mathrm{CNT}$ (solid line) and 0.25CNT (dashed line) samples. b Temperature coefficient of resistance (squares) and dimensionless sensitivity for 1CNT sample averaged \langle\rangle in frequency bands (triangles) versus temperature
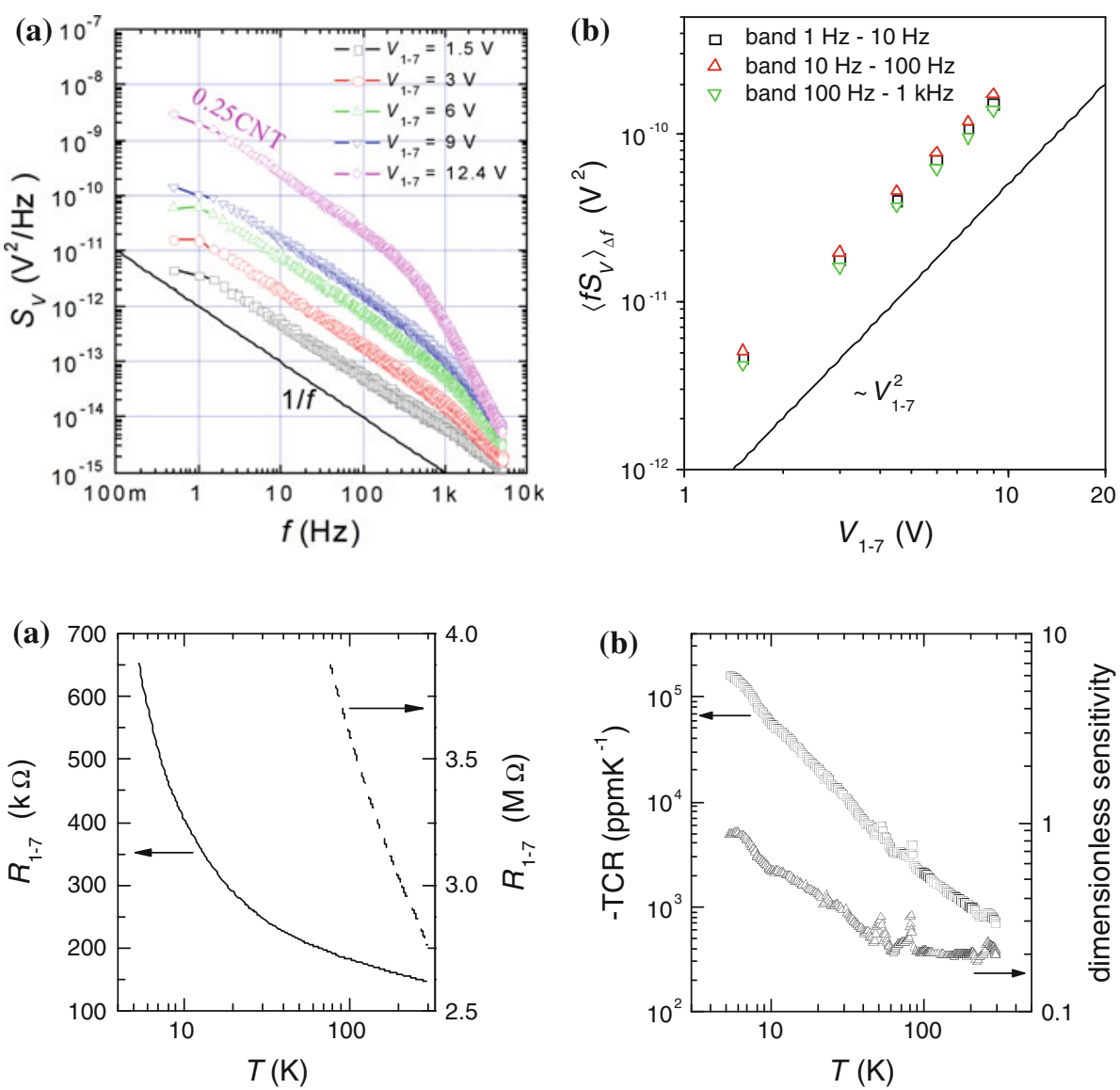

Fig. 11 a Noise intensity on terminations $1-7$ versus temperature. b Product $f_{s_{7-7}} T^{-1}$ versus frequency and temperature (noise map). Thermally activated noise sources have been revealed as streaks

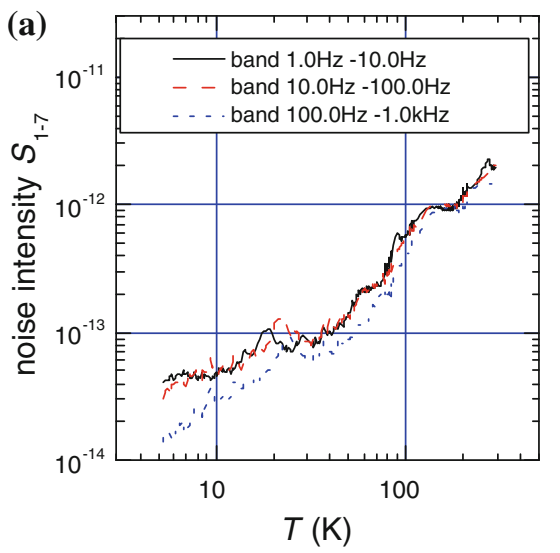

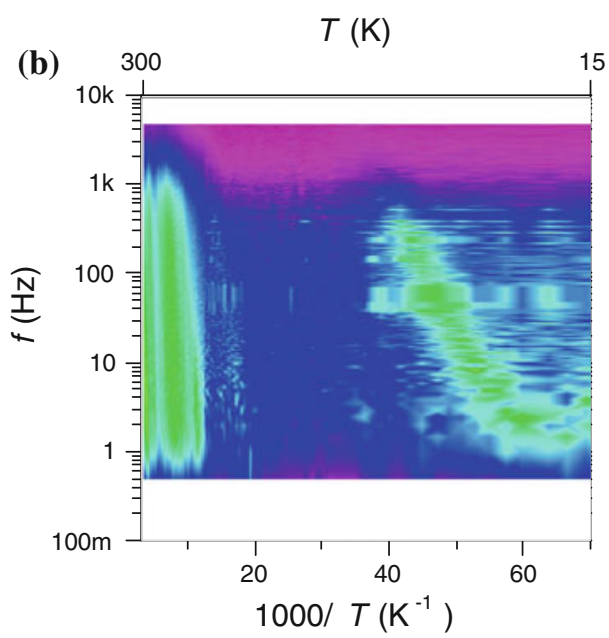

Integral measure of noise [19]

$s_{T} \equiv \frac{1}{T_{2}-T_{1}} \int_{T_{1}}^{T_{2}} \int_{f_{1}}^{f_{2}}\left(S_{V}-S_{V=0}\right) I^{-2} d f d T$

was calculated and used for comparison of noise properties of different resistive materials. The above integral was performed over frequency band from $f_{1}=10 \mathrm{~Hz}$ to $f_{2}=100 \mathrm{~Hz}$ and temperature range from $T_{1}=77 \mathrm{~K}$ to
$T_{2}=300 \mathrm{~K}$. The above limits in the integral were chosen to make possible reference to the results obtained for other resistive layers $[19,20]$. The advantage of the integral measure of noise becomes clear, when we look at the "chaotic" plot of power of resistance fluctuations $\left\langle\delta R^{2}\right\rangle$ shown in Fig. 12a. As the set of TANSs is sample specific, so the plot of $\left\langle\delta R^{2}\right\rangle$ is also different for different samples. Hence, it is impossible to use directly values of such plot for reliable comparison of noise properties of different 
Fig. 12 a power of resistance fluctuations in frequency band $10-100 \mathrm{~Hz}$ versus temperature measured on terminations $1-7$. b Integral measure of noise $s_{T}$ (circles) versus size of the sector's and linear approximation (solid line)

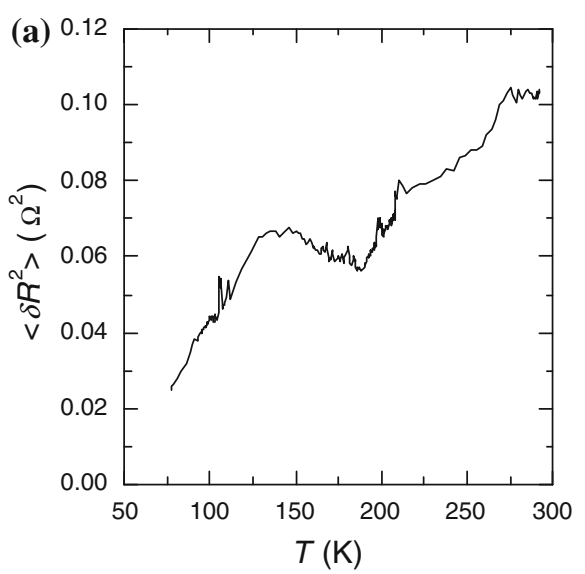

materials. In this way we arrive at the conclusion that integral measure of noise is the most appropriate parameter for noise properties comparison.

The linear approximation of $s_{T}$ versus resistor's sector size, from which $S_{V}$ was acquired (sector size is $L_{x y} / w$, where $L_{x y}$ is the length of the resistive layer spanned between terminations $\mathrm{x}-\mathrm{y}$ : 1-7, 1-6, 2-6, 3-5), gives noise intensity per square $s_{\square}$ (slope) and $s_{\text {int }}$ (see Fig. 12b), which leads to bulk noise intensity $C_{\text {bulk }} \equiv s_{\square} / R_{\square}^{2} \times \Omega_{\square}$, where $R_{\square}$-is sheet resistance and $\Omega_{\square}=w^{2} d$ is volume of the individual square. Also values of $C_{\text {bulk }}$ for polymer filled with carbon black systems presented in Fig. 13 were calculated in earlier works [24].

The calculated value $C_{\text {bulk }}=1.28 \cdot 10^{-22} \mathrm{~m}^{3}$ obtained for the resistive layer of polymer-CNT nanocomposite, with $R_{\square}=9.4 \mathrm{k} \Omega$ is close to the value $2 \cdot 10^{-22} \mathrm{~m}^{3}$ for $\mathrm{Pb} / \mathrm{Cd}$-free $\mathrm{RuO}_{2-}$ and $\mathrm{Bi}_{2} \mathrm{Ru}_{2} \mathrm{O}_{7}$-based layers $[19,20]$. However it is still an order of magnitude larger than $10^{-23} \mathrm{~m}^{3}$ obtained for

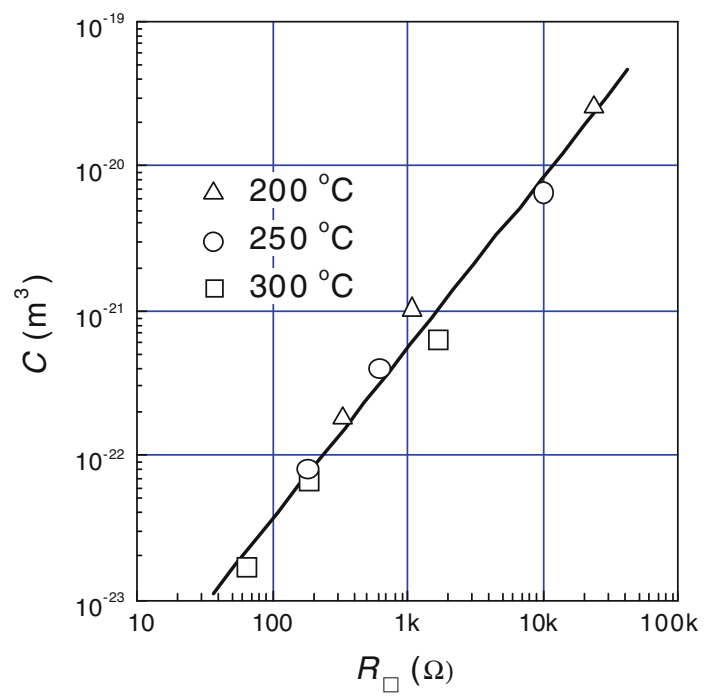

Fig. $13 C_{\text {bulk }}$ value of polymer-carbon black resistors calculated for samples measured in different temperatures
$\mathrm{Pb}$-containing $\mathrm{RuO}_{2}$-based resistors [19]. In comparison


are almost two orders higher $\left(\mathrm{C}_{\text {bulk }} \approx 3 \cdot 10^{-20} \mathrm{~m}^{3}\right)$ than for polymer-CNT resistors. It needs to be stated, that commercially available inks for cermet resistors contains noise modifiers (e.g. $\mathrm{MnO}_{2}$ ), which make the noise index of a resistor an order of magnitude lower than that for a pure model system. We believe that there are modifiers which could also reduce the noise index in polymer-CNT resistors.

As the size effect, calculated using ordinary resistance measurements, $\quad\left(R_{1-7} w / L\right) / R_{\square}=0.996$ is negligible, another noise parameter, $C_{\text {int }} \equiv w s_{\text {int }} / s_{\square}$, was involved to test the quality of the resistive-to-conductive layers interface. Relatively small value of $C_{\text {int }}=0.2 \mathrm{~mm}$ is the evidence that the resistive layer forms good interface with $\mathrm{Ag}$ contact.

\section{Conclusions}

The authors proved that it is possible to elaborate carbon nanotube thick film composite materials, which could be deposited with the screen printing technique. New thick film polymer materials containing carbon nanotubes investigated by the authors could be used to obtain various microelectronics and mechatronics systems, such as printed elastic resistors. Even though today used polymer-graphite resistors can provide wide range of resistances, they lack resilience to mechanical stress and show strong tensometric effect. The results presented in this paper demonstrate, that the elaborated polymer-nanotube layers could be good substitute for graphite resistive layers in elastic printed electronics applications, where material must sustain heavy mechanical stress. Resistance and noise measurements in cryostats have been involved. 1/f resistance noise has been observed with significantly rising intensity during temperature rise. Thermally activated noise sources of activation energies in the range $25 \mathrm{meV}-1,6 \mathrm{eV}$ have been revealed 
using low-frequency noise spectroscopy. Relatively large value of negative TCR has been obtained and calculated dimensionless sensitivity is similar to that observed in leadfree $\mathrm{RuO}_{2}$-glass and $\mathrm{Bi}_{2} \mathrm{Ru}_{2} \mathrm{O}_{7}$ resistors, as well as for cryogenic temperature sensors. Examined composites represented them self as very promising materials. More work needs to be done to improve electrical properties of the elaborated materials.

Open Access This article is distributed under the terms of the Creative Commons Attribution Noncommercial License which permits any noncommercial use, distribution, and reproduction in any medium, provided the original author(s) and source are credited.

\section{References}

1. J.H. Choi, J.H. Park, J.S. Moon, J.W. Nam, J.B. Yoo, C.Y. Park, J.H. Park, C.G. Lee, D.H. Choe, Fabrication of carbon nanotube emitter on the flexible substrat. Diam. Relat. Mater. 15, 44-48 (2006)

2. J.W. Nam, S.H. Cho, Y.C. Choi, J.S. Ha, J.H. Park, D.H. Choe, J.B. Yoo, J.H. Park, The influence of filler on the emission properties and rheology of carbon nanotube paste. Diam. Relat. Mater. 14, 2089-2093 (2005)

3. C. Berger, Y. Yi, Z.L. Wang, W.A. de Heer, Multiwalled carbon nanotubes are ballistic conductors at room temperature. Appl. Phys. A 74, 363-365 (2002)

4. R. Saito, G. Dresselhaus, M.S. Dresselhaus, Physical Properties of Carbon Nanotubes (2003), pp. 17-29

5. A. Esawi, K. Morsi, Dispersion of carbon nanotubes (CNTs) in aluminum powder. Compos. Part A 38, 646-650 (2007)

6. A.R. Boccaccini, D.R. Acevedo, G. Brusatin, P. Colombo, Borosilicate glass matrix composites containing multi-wall carbon nanotubes. J. Eur. Ceramic Soc. 25, 1515-1523 (2005)

7. T.-Y. Tsai, N.-H. Tai, I.-N. Lin, Characteristics of carbon nanotube electron field emission devices prepared by LTCC process. Diam. Relat. Mater. 13, 982-986 (2004)

8. N.S. Lee, D.S. Chung, I.T. Han, J.H. Kang, Y.S. Choi, H.Y. Kim, S.H. Park, Y.W. Jin, W.K. Yi, M.J. Yun, J.E. Jung, C.J. Lee, J.H. You, S.H. Jo, C.G. Lee, J.M. Kimb, Application of carbon nanotubes to field emission displays. Diam. Relat. Mater. 10, 265-270 (2001)

9. K. Parikh, K. Cattanach, R. Rao, D.-S. Suh, A. Wu, S.K. Manohar, Flexible vapour sensors using single walled carbon nanotubes. Sens. Actuators B 113, 55-63 (2006)

10. G. Zheng, F. Patolsky, Y. Cui, W.U. Wang, C.M. Lieber, Multiplexed electrical detection of cancer markers with nanowires sensor arrays. Nat. Biotechnol. 23, 1294-1301 (2005)
11. H. Sirringhaus, T. Kawase, R.H. Friend, T. Shimoda, M. Inbasekaran, W. Wu, E.P. Woo, High-resolution inkjet printing of all-polymer transistor circuits. Science 15 290(5499), 2123-2126 (2000)

12. A. Erdema, D.O. Ariksoysala, H. Karadeniza, P. Karaa, A. Sengonulb, A.A. Sayinerb et al., Electrochemical genomagnetic assay for the detection of hepatitis B virus DNA in polymerase chain reaction amplicons by using disposable sensor technology. Electrochem. Commun. 7, 815-820 (2005)

13. D.R. Hines, S. Mezhenny, M. Breban, E.D. Williams, Nanotransfer printing of organic and carbon nanotube thin-film transistors on plastic substrates. Appl. Phys. Lett. 86, 163101 (2005)

14. Q. Cao, Z.-T. Zhu, M.G. Lemaitre, M.-G. Xia, M. Shim, J.A. Rogers, Transparent flexible organic thin-film transistors that use printed single-walled carbon nanotube electrodes. Appl. Phys. Lett. 88(11), 113511-113513 (2006)

15. F. Axisa, A. Dittmar, G. Delhomme, Smart clothes for the monitoring in real time and conditions of physiological, emotional and sensorial reactions of human. Proc. 25th Annu. Int. Conf. IEEE 4, 3744-3747 (2004)

16. E. Carreno-Morelli, J. Yang, R. Schaller, C. Bonjour, Carbon nanotube reinforced metal matrix composites. in Proceedings of EURO PM2003, European Conference on Powder Metallurgy, Valencia, Spain, 20-22 Oct

17. Z. Wang, Q. Liu, H. Zhu, H. Liu, Y. Chen, M. Yang, Dispersing multi-walled carbon nanotubes with water-soluble block copolymers and their use as supports for metal nanoparticles. Carbon 45, 285-292 (2007)

18. J. Yang, Y. Wang, R.N. Dave, R. Pfeffer, Mixing of nano-particles by rapid expansion of high-pressure suspensions. Adv. Powder Technol. 14(4), 471-493 (2003)

19. K. Mleczko, Z. Zawiślak, A.W. Stadler, A. Kolek, A. Dziedzic, J. Cichosz, Evaluation of conductive-to-resistive layers interaction in thick-film resistors. Microelectron. Reliab. 48, 881-885 (2008)

20. A. Stadler, A. Kolek, Z. Zawiślak, K. Mleczko, M. Jakubowska, K. Kiełbasiński, A. Młożniak, Noise properties of $\mathrm{Pb} / \mathrm{Cd}$-free thick film resistors. J. Phys. D Appl. Phys. 43, 265401 (2010)

21. A.W. Stadler, Noise signal analyzer for multi-terminal devices. in Proceedings of XXXI International Conference of IMAPS Poland Chapter, Rzeszów-Krasiczyn, 23-26 Sept 2007, pp. 413-416

22. P. Ptak, A. Kolek, A.W. Stadler, K. Mleczko, Noise resolution of $\mathrm{RuO}_{2}$-based resistance thermometers. Rev. Sci. Instrum. 76, $014901(2005)$

23. A. Kolek, A.W. Stadler, P. Ptak, Z. Zawiślak, K. Mleczko, P. Szałański, D. Żak, Low-frequency $1 / f$ noise of $\mathrm{RuO}_{2}$-glass thick resistive films. J. Appl. Phys. 102, 103718 (2007)

24. A. Dziedzic, A. Kolek, 1/f noise in polymer thick-film resistors. J. Phys. D Appl. Phys. 31, 2091-2097 (1998) 